УДК 517.9

\title{
ОБОБЩЕННЫЕ ПОТЕНЦИАЛЫ ДВОЙНОГО СЛОЯ АНИЗОТРОПНОЙ ПЛОСКОЙ ТЕОРИИ УПРУГОСТИ
}

\author{
(C) 2015 г. А. П. Солдатов \\ Представлено академиком РАН Е.И. Моисеевым 24.09.2014 г.
}

Поступило 13.11.2014 г.

DOI: $10.7868 / \mathrm{S} 086956521513006 \mathrm{X}$

1. С и с е м а Л ме. В плоской анизотропной теории упругости [1] вектор смещения $u=$ $=\left(u_{1}, u_{2}\right)$ удовлетворяет эллиптической системе Ламе

$$
a_{11} u_{x x}+\left(a_{12}+a_{21}\right) u_{x y}+a_{22} u_{y y}=0
$$

с матричными коэффициентами

$$
\begin{aligned}
& a_{11}=\left(\begin{array}{ll}
\alpha_{1} & \alpha_{6} \\
\alpha_{6} & \alpha_{3}
\end{array}\right), \quad a_{12}=\left(\begin{array}{ll}
\alpha_{6} & \alpha_{4} \\
\alpha_{3} & \alpha_{5}
\end{array}\right), \\
& a_{21}=\left(\begin{array}{ll}
\alpha_{6} & \alpha_{3} \\
\alpha_{4} & \alpha_{5}
\end{array}\right), \quad a_{22}=\left(\begin{array}{ll}
\alpha_{3} & \alpha_{5} \\
\alpha_{5} & \alpha_{2}
\end{array}\right) .
\end{aligned}
$$

Элементы $\alpha_{j}$ этих матриц, называемые модулями упругости, подчиняются требованию положительной определенности матрицы третьего порядка

$$
\alpha=\left(\begin{array}{lll}
\alpha_{1} & \alpha_{4} & \alpha_{6} \\
\alpha_{4} & \alpha_{2} & \alpha_{5} \\
\alpha_{6} & \alpha_{5} & \alpha_{3}
\end{array}\right)
$$

Из (1) видно, что соотношения $v_{x}=-\left(a_{21} u_{x}+a_{22} u_{y}\right)$, $v_{y}=a_{11} u_{x}+a_{12} u_{y}$ определяют вектор-функцию $v=$ $=\left(v_{1}, v_{2}\right)$, которая называется сопряженной к решению $и$ системы Ламе. Закон Гука заключается в том, что столбцы $\sigma_{(1)}$ и $\sigma_{(2)}$ тензора напряжений $\sigma$ выражаются через частные производные функции $v$ по формулам $\sigma_{(1)}=v_{y}, \sigma_{(2)}=-v_{x}$. Заметим, что функция $V$ постоянна тогда и только тогда, когда $u$ является многочленом первой степени вида

$$
u_{1}(x, y)=\lambda_{1}-\lambda_{0} y, \quad u_{2}(x, y)=\lambda_{1}+\lambda_{0} x .
$$

Решения $u$ системы Ламе этого типа называем тривиальными.

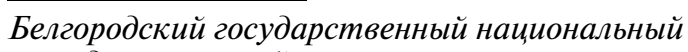
исследовательский университет

E-mail: soldatov48@gmail.com
Система Ламе (1) определяется матричным квадратным трехчленом

$$
p(z)=a_{11}+\left(a_{12}+a_{21}\right) z+a_{22} z^{2}
$$

или в явном виде

$$
p=\left(\begin{array}{cc}
p_{1} & p_{3} \\
p_{3} & p_{2}
\end{array}\right)
$$

с многочленами $p_{1}(z)=\alpha_{1}+2 \alpha_{6} z+\alpha_{3} z^{2}, p_{2}(z)=$ $=\alpha_{3}+2 \alpha_{5} z+\alpha_{2} z^{2}, p_{3}(z)=\alpha_{6}+\left(\alpha_{3}+\alpha_{4}\right) z+\alpha_{5} z^{2}$.

В силу эллиптичности характеристический многочлен $\chi=p_{1} p_{2}-p_{3}^{2}$ имеет в верхней полуплоскости два корня $v_{1}, v_{2}$, возможно совпадающие. Случаи $v_{1} \neq v_{2}$ простых корней и $v_{j}=v$ одного кратного корня указываем обозначениями, соответственно, (i) и (ii). В дальнейшем существенную роль будут играть не сами корни $v$, а их сумма и произведение, которые обозначим, соответственно, $s=v_{1}+v_{2}$ и $t=v_{1} v_{2}$ (в случае (ii) следует положить $s=2 v, t=v^{2}$ ).

Если многочлен $p_{3}=0$, т.е. $\alpha_{3}+\alpha_{4}=\alpha_{5}=\alpha_{6}=0$, система (1) распадается на два уравнения $\alpha_{1} u_{1, x x}+$ $+\alpha_{3} u_{1, y y}=0$ и $\alpha_{3} u_{2, x x}+\alpha_{2} u_{2, y y}=0$, а корни многочлена $\chi$ находятся из уравнений $p_{j}\left(v_{j}\right)=0$, т.е. $v_{1}=$ $=i \sqrt{\frac{\alpha_{1}}{\alpha_{3}}}$ и $v_{2}=i \sqrt{\frac{\alpha_{3}}{\alpha_{2}}}$. Этот случай не представляет интереса и в дальнейшем исключается из рассмотрений.

Обозначим $\mathscr{A}$ класс всех положительно определенных матриц вида (2), для которых $p_{3} \neq 0$. В этом классе выделим подмножества $\mathscr{A}_{1}$ и $\mathscr{A}_{2}$, которые определяются условием линейной независимости многочленов, соответственно, $p_{2}, p_{3}$ и $p_{1}, p_{3}$, фигурирующих в (3). Все множество $\mathscr{A}$ совпадает с объединением этих подмножеств. Вне пересечения $\mathscr{A}_{1} \cap \mathscr{A}_{2}$ модули упругости подчинены неравенству $\alpha_{3}^{2}<\alpha_{1} \alpha_{2}$. Если $\alpha \notin \mathscr{A}_{j}$, то корни многочлена $\chi$ различны и находятся из уравнений 
$\left(\alpha_{2}^{2} p_{1}-\alpha_{5}^{2} p_{2}\right)\left(v_{1}\right)=0, p_{2}\left(v_{2}\right)=0$ при $j=1$ и уравнений $p_{1}\left(v_{1}\right)=0,\left(\alpha_{3}^{2} p_{2}-\alpha_{5}^{2} p_{1}\right)\left(v_{2}\right)=0$ при $j=2$.

Упругая среда называется ортотропной, если $\alpha_{5}=\alpha_{6}=0$, в этом случае координатные прямые служат осями симметрии упругой среды и для многочленов (3) имеем более простые выражения $p_{1}(z)=\alpha_{1}+\alpha_{3} z^{2}, p_{2}(z)=\alpha_{3}+\alpha_{2} z^{2}$ и $p_{3}(z)=\left(\alpha_{3}+\alpha_{4}\right) z$. В частности, в ортотропной среде либо $\alpha \in \mathscr{A}_{1} \cap \mathscr{A}_{2}$ (напомним, что случай $p_{3}=0$ исключается). Поскольку при $\alpha_{5}=\alpha_{6}=0$ характеристическое уравнение $\chi=0$ биквадратно, его корни $v$ в верхней полуплоскости можно выразить явно с помощью положительных чисел $\rho$ и $\rho_{0}$, определяемых равенствами

$$
\rho^{2}=\sqrt{\frac{\alpha_{1}}{\alpha_{2}}}, \quad \rho_{0}^{2}=\frac{\alpha_{1} \alpha_{2}-\alpha_{4}^{2}+2 \alpha_{3}\left(\sqrt{\alpha_{1} \alpha_{2}}-\alpha_{4}\right)}{\alpha_{2} \alpha_{3}} .
$$

Заметим, что числа $\rho_{0}^{2}-4 \rho^{2}$ и $\sqrt{\alpha_{1} \alpha_{2}}-\alpha_{4}-2 \alpha_{3}$ одного знака. В обозначениях (4) для суммы и произведения корней имеют место единые выражения $s=i \rho_{0}, t=-\rho^{2}$.

Ортотропная среда называется изотропной, если дополнительно выполнены соотношения $\alpha_{1}=\alpha_{2}=2 \alpha_{3}+\alpha_{4}$. В этом случае $\alpha_{1}>\alpha_{3}$, так что $\kappa=\frac{\alpha_{1}+\alpha_{3}}{\alpha_{1}-\alpha_{3}}>1$. В рассматриваемом случае характеристическое уравнение имеет кратный корень $v=i$.

Исходя из двух типов корней характеристического уравнения, положим

$$
\text { (i) } J=\left(\begin{array}{cc}
v_{1} & 0 \\
0 & v_{2}
\end{array}\right), \quad \text { (ii) } J=\left(\begin{array}{ll}
v & 1 \\
0 & v
\end{array}\right) \text {. }
$$

Л е м м а 1. Для любого $\alpha \in \mathscr{A}_{j}, j=1,2$, существует обратимая матрица $b \in \mathbb{C}^{2 \times 2}$, которая непрерывно зависит от $\alpha$ и удовлетворяет матричному уравнению $a_{11} b+\left(a_{12}+a_{21}\right) b J+a_{22} b J^{2}=0, n p u-$ чем связанная с ней матрица $c=-\left(a_{21} b+a_{22} b J\right)$ также обратима. В случае $\alpha \in \mathscr{A}_{0}$ можно положить $b=1$.

Если матрииа $\tilde{b}$ также является решением этого уравнения, то $\tilde{b}=b d$, где матрица $d$ коммуmupyem с $J$.

2. Первая и вторая краевые задачи в классах Харди. Рассмотрим систему Ламе в области $D$ комплексной плоскости, ограниченной ляпуновским контуром $\Gamma \in C^{1, v}, 0<v<1$. Эта область может быть как конечной (т.е. лежать внутри некоторого круга), так и бесконечной (т.е. содержать внешность некоторого круга). В случае бесконечной области все простые контуры, составляющие $\Gamma$, равноправны. Если же область $D$ конечна, то один из этих контуров охватывает все остальные, его называем внешним контуром. Удобно тип области $D$ указывать сигнатурой к $(D)$, принимающей значения 1 , если эта область конечна, и 0 в противном случае. В дальнейшем в случае $\kappa(D)=0$ бесконечной области на градиент решения $u$ системы (1) накладывается условие $\left|u_{x}(z)\right|+\left|u_{y}(z)\right|=O\left(|z|^{-2}\right)$ при $z \longrightarrow \infty$, в частности, существует предел $u(\infty)=\lim u(z)$ на бесконечности. Из этого условия следует, что в случае бесконечной области тривиальными решениями могут быть только постоянные векторы.

Как известно [2], класс Харди $h^{p}(D)$ гармонических функций вводится аналогично случаю аналитических функций. Этот класс можно также ввести следующим эквивалентным способом. Условимся под диффеоморфным граничным вложением $\omega: K \rightarrow \bar{D}$ квадрата $K=\{(r, s), 0 \leq r, s \leq 1\}$ в замкнутую область $\bar{D}$ понимать взаимно однозначную функцию $\omega \in C^{1, v}(K)$ со значениями в $\bar{D} \subseteq \mathbb{C}$, для которой векторы $\omega_{r}$ и $\omega_{s}$ всюду линейно независимы и $\omega(0, s) \in \Gamma, \omega(r, s) \in D$ при $0<r \leq 1$. Тогда пространство $h^{p}(D)$ можно определить как класс всех гармонических функций $u(z), z \in D$, которые допускают почти всюду на $Г$ угловые предельные значения $u^{+}$и удовлетворяют условию

$$
\sup _{0<r \leq 1} \int_{0}^{1}|(u \circ \omega)(r, s)|^{p} d s<\infty
$$

для любого диффеоморфного граничного вложения $\omega: K \rightarrow \bar{D}$. Последнее условие влечет $u^{+} \in L^{p}(\Gamma)$, причем пространство $h^{p}$ банахово относительно нормы $|u|=\left|u^{+}\right|_{L^{p}}$.

Это определение можно распространить на любые функции $w \in C(D)$, его ниже используем для решений $w=u$ системы Ламе и сопряженных к ним функций $w=v$. Пусть граница Г области $D$ состоит из простых контуров $\Gamma_{1}, \ldots, \Gamma_{m}$, причем в случае $\kappa(D)=1$ контур $\Gamma_{m}$ является внешним. Тогда при $m \geq 2$ функция $v$, сопряженная к решению $u$ системы Ламе, вообще говоря, будет многозначной, оставаясь однозначной для своих частных производных. При обходе контуров $\Gamma_{j}, 1 \leq j \leq m-1$, она получает конечные приращения. Можно выделить такое конечномерное пространство $U(D) \subseteq$ $\subseteq C^{\infty}(\bar{D})$ размерности $2(m-1)$ решений $u_{0}$ системы Ламе, что сопряженные к ним функции многозначны и любое решение $u$ этой системы единственным образом представляется в виде $u=u_{0}+u_{1}$, $u_{0} \in U(D)$, где функция, сопряженная к $u_{1}$, однозначна. Совершенно аналогично обстоит дело и с однозначными функциями, сопряженными к многозначным решениям системы Ламе. Соответствующее пространство для этих функций обозначаем $V(D)$. С помощью указанных разло- 
жений пространство Харди $h^{p}$ естественным образом распространяется и на многозначные функции.

Те о ре м а 1. Пространство $h^{p}(D), p>1$, решений $w=$ и системы Ламе и аналогичное пространство сопряженных к ним функций $w=v$ банахово относительно нормы $|w|=|w|_{L^{p}}$, причем $и \in h^{p}$ равносильно $\mathrm{V} \in h^{p}$.

Как известно [3], основные краевые условия для системы Ламе состоят в задании на граничном контуре либо вектора смещений $u^{+}=f$ (задача Дирихле), либо нормальной компоненты $\sigma^{+} n=$ $=\sigma_{(1)}^{+} n_{1}+\sigma_{(2)}^{+} n_{2}$ тензора напряжения $\sigma$, где $n=n_{1}+$ $+i n_{2}$ есть единичная внешняя нормаль на Г (задача Неймана). Согласно закону Гука последнее краевое условие можем записать в форме $\left(V^{+}\right)^{\prime}=g$ для касательной производной граничного значения $V^{+}$сопряженной функции.

С точки зрения общих сильно эллиптических систем вопросы разрешимости задач Дирихле и Неймана для системы Ламе в гёльдеровских и соболевских пространствах хорошо изучены [4]. В частности, задача Дирихле однозначно разрешима в этих пространствах, а задача Неймана фредгольмова индекса нуль. Более точно, все решения однородной задачи тривиальны, а условие ортогональности

$$
\int_{\Gamma} g u_{0}^{+}|d t|=0
$$

правой части $g$ неоднородной задачи тривиальным решениям $u_{0}$ необходимы и достаточны для ее разрешимости. Напомним, что в случае бесконечной области $D$ тривиальными решениями являются постоянные векторы $u_{0}=\xi \in \mathbb{R}^{2}$, а при $\kappa(D)=1$ число условий ортогональности (5) равно трем.

Поскольку функция $V$ в краевом условии $\left(V^{+}\right)^{\prime}=$ $=g$, вообе говоря, многозначна, проинтегрировать это равенство в классе непрерывных функций нельзя. Однако с помощью указанных выше разложений можно перейти к задаче Дирихле $V^{+}=f$ для однозначной функции $v$, сопряженной к многозначному решению системы Ламе. В определенном смысле полученная задача будет эквивалентной задаче Неймана. Тем самым открывается возможность рассмотрения задач Дирихле для решений (1) и сопряженных к ним функций в классах классах Харди $h^{p}(D)$. При этом условия ортогональности (5) по отношению к последней задаче в случае $(D)=0$ бесконечной области снимаются, а в случае $\kappa(D)=1$ они переходят в одно условие

$$
\int_{\Gamma} f(t) n(t)|d t|=0
$$

где подынтегральное выражение понимается как скалярное произведение $f_{1}(t)$ и вектора нормали $n=\left(n_{1}, n_{2}\right)$ в точке $t \in \Gamma$ в $\mathbb{R}^{2}$.

Те о р е м а 2. Пусть область D ограничена кон-

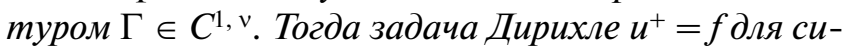
стемы Ламе в классе $h^{p}(D), p>1$, однозначно разрешима, а задача Дирихле $\mathrm{V}^{+}=f$ фредгольмова и ее индекс равен -к(D). Более точно, однородная задача имеет только нулевое решение, а неоднородная задача при $\kappa(D)=0$ безусловно разрешима, а при $\kappa(D)=1$ она разрешима тогда и только тогда, когда ее правая часть удовлетворяет условию ортогональности (6).

Если правая часть $f$ этих задач принадлежит классу $C^{\mu}(\Gamma), 0<\mu<v$, то и любое их решение принадлежст $C^{\mu}(\bar{D})$. Аналогичное утверждение справедливо и по отношению к классам $C^{1, \mu}$.

3. Обобщенны е потенциалы двой ного слоя. В исследованиях краевых задач анизотропной плоской упругости в классических пространствах Гёльдера и Соболева можно выделить два основных направления. Первое из них состоит в использовании аналитических функций по аналогии с формулами Колосова-Мусхелишвили [3] в изотропном случае. Это направление представлено работами С.Г. Лехницкого, Г.Н. Савина, С.Г. Михлина и др. (см., например, $[5,6]$.$) Второе направление основано на примене-$ нии классического метода потенциала, оно развивалось В.Д. Купрадзе [1], М.О. Башелейшвили и др.

Достаточно широкое распространение получило также использование вместо аналитических функций решений эллиптических систем первого порядка с постоянными коэффициентами, являющихся частным случаем обобщенных аналитических функций И.Н. Векуа (см., например, [7-9]). Представление общего решения системы Ламе через так называемые функции, аналитические по Дуглису, полученное в [9] (см. также [10]), было использовано [11] для введения обобщенных потенциалов двойного слоя. В данной работе приведены окончательные результаты в смысле явного описания этих потенциалов только через модули упругости и простейшие симметричные комбинации корней характеристического уравнения в общем анизотропном случае. С помощью этих потенциалов, по-видимому, впервые удается распространить разрешимость задач Дирихле и Неймана на класс $C(\bar{D})$ в рамках общего теоретико-функционального подхода, развитого в [12].

Интеграл типа Коши для аналитических функций с вещественной плотностью $\varphi$ можно записать в виде $P \varphi-i Q \varphi c$ интегралом

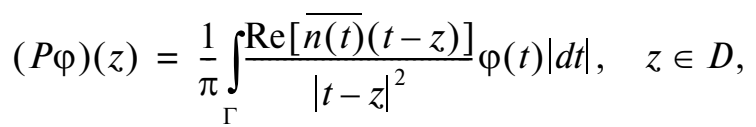


где $|d t|$ означает элемент длины дуги, $n=n_{1}+i n_{2}$ есть единичный вектор внешней нормали и $d t=$ $=i n(t)|d t|$. Интеграл $Q \varphi$ определяется аналогично по отношению к $\operatorname{Im}[\overline{n(t)}(t-z)]$.

Хорошо известно [2], что для $\varphi \in L^{p}(\Gamma), p>1$, аналитическая функция $\phi(z)$, определяемая интегралом типа Коши, принадлежит классу Харди $H^{p}(D)$ и допускает почти всюду на Г угловые предельные значения $\phi^{+}\left(t_{0}\right)$, для которых справедлива формула Сохоцкого-Племеля $\phi^{+}=\varphi+P^{*} \varphi-i Q^{*} \varphi$, где * означает, что интегралы берутся в граничных точках $t_{0} \in \Gamma$. Таким образом, $(P \varphi)^{+}=\varphi+P^{*} \varphi$, $(Q \varphi)^{+}=Q^{*} \varphi$. Напомним, что контур Г предполагается ляпуновским и принадлежит $C^{1, v}$, поэтому ядро интеграла $P^{*} \varphi$ имеет слабую особенность. Интеграл $\left(Q^{*} \varphi\right)\left(t_{0}\right)$ сингулярный и понимается в смысле главного значения по Коши. Отсюда, в частности, следует, что вещественные функции $P \varphi$ и $Q \varphi$ принадлежат классу Харди $h^{p}(D)$ гармонических функций. В случае $\varphi \in C^{\mu}(\Gamma)$ или $\varphi \in$ $\in C^{1, \mu}(\Gamma), 0<\mu<v$, функции $Р \varphi$ и $Q \varphi$ принадлежат аналогичному классу в замкнутой области $\bar{D}$. Кроме того, в случае $\varphi \in C(\Gamma)$ функция $P \varphi \in$ $\in C(\bar{D})$, а $Q \varphi \in h^{p}(D)$ для любого $p>1$.

Интеграл Р९ представляет собой классический потенциал двойного слоя для уравнения Лапласа. По аналогии с ним в обозначениях леммы 1 введем интегралы

$$
\begin{gathered}
\left(P_{k r} \varphi\right)(z)=\frac{1}{\pi} \int_{\Gamma}^{\operatorname{Re}[\overline{n(t)}(t-z)]} H_{k r}(t-z) \varphi(t)|d t|, \\
|t-z|^{2} \\
z \in D,
\end{gathered}
$$

с плотностью $\varphi=\left(\varphi_{1}, \varphi_{2}\right) \in L^{p}(\Gamma), p>1$ и матричными множителями $H_{11}(\xi)=\operatorname{Im}\left[b h(\xi, J) b^{-1}\right]$, $H_{22}(\xi)=\operatorname{Im}\left[\operatorname{ch}(\xi, J) c^{-1}\right], H_{21}(\xi)=\operatorname{Im}\left[\operatorname{ch}(\xi, J) b^{-1}\right]$, $H_{12}(\xi)=\operatorname{Im}\left[b h(\xi, J) c^{-1}\right]$, где положено $h(\xi, J)=$ $=\left(-\xi_{2}+i \xi_{1} J\right)\left(\xi_{1}+\xi_{2} J\right)^{-1}$. В силу леммы 1 эти матрицы не зависят от выбора матрицы $b$ и связанной с ней матрицы $c$.

Теорема 3. Каждая пара равенств $и=$ $=P_{11} \varphi, v=-\left[\operatorname{Im}\left(c b^{-1}\right)\right] Q \varphi+P_{21} \varphi u v=P_{22} \varphi, u=$ $=-\left[\operatorname{Im}\left(b c^{-1}\right)\right] Q \varphi+P_{12} \varphi$ определяет решение $u$ системы Ламе и сопряженную к ней функцию $v$.

В соответствии с этой теоремой интегралы $P_{11} \varphi$ и $P_{22} \varphi$ называем обобщенными потенциалами двойного слоя для решений системы Ламе и сопряженных к ним функциям. Пусть интегралы $\left(P_{k r}^{*} \varphi\right)\left(t_{0}\right)$ определяются аналогично (7) по отношению к $z=t_{0} \in \Gamma$. Как и в случае интеграла $P \varphi$, ядра $|t-z|^{-2} \operatorname{Re}\left[\overline{n(t)}\left(t-t_{0}\right)\right] H_{k r}\left(t-t_{0}\right)$ имеют слабые особенности и, следовательно, операторы $P_{k r}^{*}$ компактны в пространствах $C(\Gamma)$ и $L^{p}(\Gamma)$.

Л ем ма 2. Интегральные операторы $P_{k r}$ ограничены $L^{p}(\Gamma) \rightarrow h^{p}(D), 1<p<\infty$, и справедливы формулы $\left(P_{11} \varphi\right)^{+}=\varphi+P_{11}^{*} \varphi,\left(P_{21} \varphi\right)^{+}=\left[\operatorname{Re}\left(c b^{-1}\right)\right] \varphi+P_{21}^{*} \varphi u$ $\left(P_{22} \varphi\right)^{+}=\varphi+P_{22}^{*} \varphi,\left(P_{12} \varphi\right)^{+}=\left[\operatorname{Re}\left(b c^{-1}\right)\right] \varphi+P_{12}^{*} \varphi$.

Эти операторы такжсе ограничены $X(\Gamma) \rightarrow X(\bar{D})$, где Хозначает любой из символов $C, C^{\mu}, C^{1, \mu}, 0<\mu<v$, причем операторы $P_{k r}^{*}$ компактны в X(Г).

Согласно теореме 3 , функция $v$, сопряженная к решению $u=P_{11} \varphi$, должна быть однозначной. В частности, решения $u \in U(D)$ системы Ламе раздела 2 не могут быть представлены потенциалом двойного слоя $P_{11} \varphi$. В случае бесконечной области $D$ это относится и к постоянным векторам $u=\xi \in \mathbb{R}^{2}$. Аналогично обстоит дело и с представлением однозначных сопряженных функций $V \in$ $\in V(D)$ к (вообще говоря, многозначным) решениям системы (1).

Те о р е м а 4. Пусть область D ограничена контуром $\Gamma \in C^{1, v}$. Тогда операторы $P_{k k}$ фредгольмовы $L^{p}(\Gamma) \rightarrow H^{p}(D)$, причем $\operatorname{ker} P_{11} \subseteq \operatorname{ker} P_{22} u \operatorname{ind} P_{11}=0$, $\operatorname{dim}\left(\operatorname{ker} P_{11}\right)=2 m-2 \kappa(D) u \operatorname{ind} P_{22}=1, \operatorname{dim}\left(\operatorname{ker} P_{22}\right)=$ $=3 m-2 \kappa(D)$, где т есть число связных компонент контура $\Gamma$.

Ядро ker $P_{11}$ состоит из постоянных на контурах $\Gamma_{k}$ вектор-функций $\varphi=\left(\varphi_{1}, \varphi_{2}\right)$, которые при $\kappa(D)=1$ обращаются в нуль на внешнем контуре, $a$ образ $\mathrm{im} P_{11}$ coстоит из всех функций и $\in H^{p}(D)$, сопряженная функция к которым однозначна в области $D$ и которые при $\kappa(D)=0$ исчезают на $\infty$.

Основываясь на теореме 2 , ядро $\operatorname{ker} P_{22}$ можно также точно описать, в частности, оно содержится в $C^{1, \mu}(\bar{D})$ для любого $0<\mu<v$. Теорема 4 позволяет свести задачи Дирихле для решений системы (1) или сопряженных к ним функций к эквивалентным системам интегральных уравнений Фредгольма на границе Г.

Матрицы $H_{k r}(\xi)$ в (7) можно описать в явном виде непосредственно в терминах элементов матрицы (2) и комбинаций $s=v_{1}+v_{2}, t=v_{1} v_{2}$ корней характеристического уравнения.

Работа выполнена при поддержке Международного проекта (0113РК01031) Министерства образования и науки Республики Казахстан.

\section{СПИСОК ЛИТЕРАТУРЫ}

1. Купрадзе В.Д. Методы потенциала в теории упругости. М.: Физматгиз, 1963. 664 с.

2. Голузин Г.М. Геометрическая теория функций комплексного переменного. М.: Наука, 1972. 628 с. 
3. Мусхелишвили Н.И. Некоторые основные задачи математической теории упругости. М., 1966. 709 с.

4. Фикера $Г$. Теоремы существования в теории упругости. М.: Мир, 1974. 160 с.

5. Лехницкий С.Г. Теория упругости анизотропного тела. М.: Л., 1950. 417 с.

6. England A.H. Complex Variable Methods in Elasticity. L.; N.Y.; Sydney, Tokyo: Intersci., 1971. 197 p.

7. Begehr H., Lin Wei. In: Partial Diifferential Equations with Real Analysis. Longman Sci. and Techn., 1992. P. 219-239.
8. Gilbert R.P., Lin Wei // J. Elasticity. 1985. V. 15. P. 143154.

9. Солдатов А.П. // ДАН. 2002. Т. 385. № 2. С. 163167.

10. Soldatov A.P. // Anal. Oldenbourg Wiss. 2010. V. 20. № 2. P. $107-117$

11. Soldatov A.P. // Proc. Appl. Math. and Mech. 2007. V. 7. № 1. P. 2040083-2040084.

12. Солдатов А.П. // Дифференц. уравнения. 2003. Т. 39. № 5. С. 674-686. 